\title{
TRATAMIENTO MÉDICO-CONSERVADOR VERSUS INTERVENCIONISTA DEL REFLUJO VESICOURETERAL PRIMARIO EN EL NIÑO
}

\author{
Joaquín Escribano Subias' y Gloria Fraga Rodríguez². \\ Unidad de Nefrología Pediátrica. Hospital Universitario Sant Joan. Universidad Rovira i Virgili. Reus. Tarragona. España. \\ ¿Unidad de Nefrología Pediátrica. Hospital de la Santa Creu i Sant Pau. Barcelona. España.
}

\begin{abstract}
Resumen.- OBJETIVO: Valorar la estrategia de tratamiento del reflujo vesico-ureteral primario (RVU) en el niño, teniendo en cuenta su repercusión sobre la aparición de infecciones urinarias, daño parenquimatoso renal y alteración de la función renal.
\end{abstract}

MÉTODOS/RESULTADO: Se realiza una búsqueda en MEDLINE de la literatura, seleccionando ensayos clínicos y metaanálisis que comparen el tratamiento intervencionista lquirúrgico o endoscópico) y conservador (profilaxis antibiótica o medidas higiénicas) del RVU en el niño. Los ECA seleccionados agrupan un total de 820 niños afectos de RVU y aleatorizados a recibir tratamiento médico o quirúrgico. Los resultados muestran que no existen diferencias en el número global de infecciones urinarias, aunque los niños operados padecen menos pielonefritis (RR 0.43). Ambos grupos presentaron igual número de nuevas cicatrices renales y de progresión de las ya existentes. No se encontraron diferencias en la afectación de la función renal, valorada como aparición de hipertensión arterial o disminución del filtrado glomerular.

CONCLUSIONES. El tratamiento quirúrgico del RVU no aporta ventajas sobre el conservador en cuanto a preservación del funcionalismo renal o aparición de nuevas cicatrices. El tratamiento conservador sería la elección inicial prioritaria.

Palabras clave: Reflujo vesicoureteral. Infección urinaria. Riñón. Metanálisis. Niño.

Summary.- OBJECTIVES: To assess the efficacy of me-dical and surgical treatment of vesicoureteral reflux (VUR) in children using recurrence of urinary tract infectio-ns, renal scarring and renal function as end-points.

METHODS/RESULTS: We performed a MEDLINE search for articles. We selected only randomized clinical trials and meta-analysis that analyzed medical versus sur-gical treatment of VUR in children. A total of 820 patients were included in all studies. We found no statistatically significant differences between surgically and medically treated patients in terms of scarring, kidney function or recurrence of urinary tract infections. There was only a significant decrease in the frequency of febrile UTI in patients who were surgically corrected, compared with those receiving antibiotics alone (RR 0.43).

CONCLUSIONS: We found no clinically significant differences between surgical and medical treatment for VUR in terms of kidney function or renal scarring. We suggest that a child with UTI and VUR should be treated conservatively at first.

Keywords: Vesicoureteral reflux. Urinary tract infection. Kidney. Meta-analysis. Child. 


\section{CONSIDERACIONES INICIALES}

El reflujo vésico-ureteral (RVU) es una malformación congénita que condiciona el paso retrógrado de la orina de la vejiga al tracto urinario superior. Se debe a una anomalía madurativa del mecanismo valvular de la unión ureterovesical y puede resolverse de forma espontánea con el paso de los años. Dependiendo del volumen de orina refluido y de las alteraciones morfológicas que produce en las vías urinarias, se clasifica en cinco grados crecientes, considerando los grados I-II como no dilatados y los grados III-V como dilatados (1). Es la malformación urológica más común en el niño y su incidencia se estima en el $1 \%$ de todos los recién nacidos, presentando una clara predisposición familiar y por tanto un posible origen genético multifactorial.

Al diagnóstico de RVU suele llegarse a partir de dos escenarios clínicos diferentes: recién nacidos con historia de hidronefrosis congénita diagnóstica por ecografía prenatal o niños con historia de infección urinaria.

En el caso del diagnóstico prenatal de hidronefrosis fetal, hasta un $40 \%$ de estos niños pueden presentar reflujo. Estos pacientes, generalmente de sexo masculino, presentan reflujos de grado elevado, que suelen evolucionar de forma espontánea a la resolución y donde hasta un $30 \%$ tiene asociada displasia o hipoplasia renal congénita, lo cual pone de relieve daño renal permanente en ausencia de infección. Aunque también presentan tendencia a padecer infecciones urinarias, no está claro que éstas modifiquen el daño establecido, ni el pronóstico a largo plazo $(2,3)$.

En los casos de diagnóstico postnatal asociado clínicamente a la existencia de infección del tracto urinario, se han detectado RVU hasta en un $20-30 \%$ de los niños afectos y de sus familiares de primer grado. Predomina en el sexo femenino $(4,5)$.

Posiblemente ambos tipos de RVU, respondan a entidades nosológicas diferentes, con origen genético diverso (6). Nosotros nos ocuparemos del RVU primario asociado a infección urinaria y diagnóstico postnatal, por ser el más prevalente y sobre el que se tiene mayor experiencia clínica.

El RVU se ha asociado con el riesgo de padecer infecciones urinarias de repetición. En el caso de infecciones febriles se puede provocar lesión estructural en el parénquima renal, con aparición de cicatrices que pueden llegar a comprometer su funcionalismo, ocasionando hipertensión arterial e incluso insuficiencia renal crónica. A mayor grado de RVU, mayor riesgo de aparición de cicatrices renales y mayor extensión de las mismas (7). Por otra parte es bien conocida la tendencia natural a la resolución espontánea de la mayoría de los RVU no dilatados (8).

Teniendo en cuenta estas consideraciones el abordaje tradicional del RVU ha postulado dos opciones fundamentales: la corrección funcional-anatómica del reflujo mediante una técnica intervencionista (quirúrgica o endoscópica) o el tratamiento conservador basado en medidas higiénicas generales y profilaxis antibiótica en espera de la resolución espontánea del problema.

Ambas estrategias de tratamiento han ponderado para valorar su eficacia, su capacidad para disminuir el número de infecciones urinarias, y lo que es más importante su capacidad de protección del parénquima renal y secundariamente de la función renal a largo plazo.

Aunque en la literatura podemos encontrar múltiples estudios de cohorte y casuísticas muy amplias que contrastan ambas estrategias, generalmente en análisis históricos de series, se encuentran pocos trabajos que aborden el problema desde estudios científicos de diseño riguroso. Nosotros hemos intentado seleccionar este tipo de estudios (metaanálisis o ensayos clínicos randomizados) para dar respuesta a la disyuntiva terapéutica planteada con suficiente garantía de que los resultados obtenidos ofrezcan evidencia científica de calidad.

Dado que el riesgo de infección urinaria, de daño renal y de evolución natural de la patología está muy influenciado por el grado de reflujo, analizaremos por separado los reflujos dilatados (grado III -V) y no dilatados (grado I -II).

\section{ACTITUD ANTE LOS RVU NO DILATADOS}

La resolución espontánea del RVU no dilatado es muy amplia. Entre el $75-100 \%$ de los niños con RVU de bajo grado lo corrigen de forma espontánea, con una media en años para la corrección de 2,7 años en los de grado I y 3,1 en los de grado II, independientemente de la edad del diagnóstico o de si son uni o bilaterales $(9,12)$.

El grado de daño renal en los reflujos no dilatados es bajo. La incidencia de aparición de cicatrices renales en niños afectos de RVU no dilatado, tras un episodio de pielonefritis, muestra cifras similares a las de los niños con pielonefritis sin RVU, y siempre muy por debajo de los pacientes con RVU dilatado $(7,10,11)$.

En estudios de cohorte en niños con IRC atribuible exclusivamente a RVU, se aprecia como los niños con reflujo de bajo grado en el momento del diagnóstico representan sólo el $7 \%$ de la muestra, correspondiendo el $93 \%$ a los pacientes con unidades renales con RVU dilatado (grados III-V). (8)

La elevada tendencia a la resolución espontánea, junto con la baja tasa de infección y daño renal, ha aconsejado tratar de forma conservadora a estos pacientes prácticamente de forma universal. Las dos grandes conferencias de consenso sobre el manejo del RVU primario en el niño, recogidas en la literatura, de las sociedades Americana de Urología y del panel de expertos de la sociedad Sueca de Medicina, recomendaban esta actitud ante los reflujos de bajo grado (ver Tablas I y II) $(12,13)$. La actitud médica conservadora puede llevar asociado el uso de profilaxis antibiótica o la promoción de simples medidas higiénicas (micciones frecuentes, ingesta elevada de agua y limpieza perineal). 


\section{ACTITUD ANTE LOS RVU DILATADOS}

Realmente la controversia surge en el enfoque del tratamiento de los pacientes con RVU dilatado, ya que en estos pacientes convergen todos los factores de riesgo para desencadenar nefropatía de reflujo y posible insuficiencia renal crónica. El abordaje intervencionista, por medio de una corrección quirúrgica o endoscópica transuretral, pretende subsanar el defecto anatómico funcional, impidiendo el paso retrógrado de la orina de la vejiga al uréter y de esta forma disminuir el riesgo de infección urinaria febril y de daño renal secundario. Se trataría de una estrategia reparadora-curativa, eliminando el problema de base de una forma eficaz y esperando alcanzar resultados correctos a largo plazo. Desgraciadamente el daño renal establecido no se ve modificado por esta estrategia, ni su éxito funcional garantiza que no aparezcan nuevas infecciones urinarias febriles (14). Estos últimos hechos hicieron pensar que una conducta expectante, con el control de las infecciones intercurrentes y la toma de medidas profilácticas higiénicas - farmacológicas, podría ser tan eficaz para preservar la función renal y menos agresiva para el paciente que la conducta anterior.

Para intentar dar respuesta a esta controversia se diseñaron a lo largo de los años 90 diversos ensayos clínicos de gran envergadura que han intentado acotar la incertidumbre de la actitud terapéutica correcta. De ellos hemos seleccionado aquellos estudios que mostraban una metodología científicamente más rigurosa y que permiten alcanzar elevados niveles de evidencia cientí- fica en sus conclusiones. El estudio más importante es la actualización publicada en la Cochrane Library (16) de la revisión sistemática publicada por Wheeler originalmente en 2003 (15). En este metaanálisis, elaborado con excelente metodología, se analizan diez ensayos clínicos que incluían 964 niños con RVU primario. De este estudio nos centraremos en el bloque de siete ensayos clínicos que comparan tratamiento quirúrgico y conservador. Entre ellos destaca el Internacional Reflux Study in Children (IRSC) $(17,18,19)$, estudio multicéntrico internacional, con una rama europea y otra norteamericana, que incluyó 612 niños con RVU dilatado (grados III-IV), en un estudio aleatorizado a recibir corrección quirúrgica o tratamiento antibiótico prolongado, y un seguimiento a largo plazo. En esta revisión sistemática se incluyen resultados de hasta 5 años de seguimiento del IRSC. Otro estudio destacable es el Birmingham Reflux Study (20), realizado en Gran Bretaña, incluyendo a 161 niños de RVU dilatado, y que recibieron tratamiento quirúrgico o antibiótico, analizando los resultados tras dos años de seguimiento.

Para completar los datos aportados en le revisión incluiremos los resultados finales del IRSC (rama europea), tras 10 años de seguimiento, publicados recientemente por Jodal, y que pueden complementar los resultados expresados anteriormente (21).

Analizaremos ambas opciones terapéuticas con respecto a las más relevantes variables clínicas pronósticas (Tabla III).

TABLA I. RECOMENDACIONES ESQUEMÁTICAS DE LA ASOCIACIÓN AMERICANA DE UROLOGÍA PARA EL TRATAMIENTO DEL RVU EN NIÑOS TENIENDO EN CUENTA SU EDAD Y GRADO DE REFLUJO (12).

\begin{tabular}{|c|c|c|c|}
\hline $\begin{array}{c}\text { Edad del paciente } \\
\text { (años) }\end{array}$ & Grado del RVU & Tratamiento & Comentarios \\
\hline$<1$ & Cualquier grado & Profilaxis antibiótica & \\
\hline 1 a 5 & I y II & Profilaxis antibiótica & \\
\hline 1 a 5 & III y IV & Profilaxis antibiótica & $\begin{array}{l}\text { Si cicatrices renales o RVU } \\
\text { bilateral, cirugía opcional }\end{array}$ \\
\hline 1 a 5 & V & Profilaxis antibiótica & $\begin{array}{l}\text { Si cicatrices renales o RVU } \\
\text { bilateral, cirugía opcional }\end{array}$ \\
\hline$\geq 6$ & I y II & Profilaxis antibiótica & $\begin{array}{l}\text { Si cicatrices renales o RVU } \\
\text { bilateral, cirugía opcional }\end{array}$ \\
\hline$\geq 6$ & III y IV & Profilaxis antibiótica & $\begin{array}{l}\text { Si cicatrices renales o RVU } \\
\text { bilateral, cirugía opcional }\end{array}$ \\
\hline$\geq 6$ & V & Corrección quirúrgica & \\
\hline
\end{tabular}




\section{Infecciones urinarias}

El primer punto a considerar es si ambas opciones consiguen resultados diferentes, ante el primer paso nosológico de la nefropatía de reflujo, como es la aparición de pielonefritis. Al analizar los datos del metaanálisis no se encuentran diferencias en el número total de infecciones urinarias recurrentes entre ambas estrategias de tratamiento, ni a los 2, 5 ó 10 años de seguimiento. Un 38,4\% de 125 niños con corrección quirúrgica y profilaxis antibiótica presentó alguna infección urinaria durante un periodo de seguimiento de 10 años, frente a un $37,7 \%$ de 127 niños con tratamiento exclusivo con antibióticos (21). Existe por tanto una fuerte evidencia científica de que el número global de infecciones urinarias presentadas por estos pacientes tras largos periodos de observación, es independiente del tratamiento del reflujo realizado. Datos similares aparecen reflejados en un nuevo metaanálisis que incluye algún nuevo ensayo clínico (22).

Sin embargo los niños con corrección quirúrgica del reflujo acompañados de tratamiento antibiótico profiláctico presentan menos episodios de pielonefritis en el seguimiento durante 5 años (20 casos en 211 niños) que los niños sólo con profilaxis antibiótica (48 casos en 218 niños) (RR $0.43[0,27,0,70])$ (16). Datos similares aparecen en seguimientos más prolongados (21). De los datos de este metaanálisis se puede deducir que asumiendo una incidencia de un $20 \%$ de ITU en niños con RVU durante 5 años de seguimiento, serían necesarias 9 intervenciones quirúrgicas para prevenir una pielonefritis. En este aspecto radicaría la principal ventaja de la actitud intervencionista y que puede justificar su indicación en situaciones de infecciones urinarias de repetición.

\section{Cicatrices renales}

Este parece el punto clave del análisis, ya que ambas estrategias intentan minimizar el daño renal adquirido y preservar así la funcionalidad. La mayor incidencia de pielonefritis entre los niños no operados, hacía presuponer que la opción intervencionista conseguiría reducir la lesión renal, sin embargo no hay diferencias en el riesgo de aparición de nuevas lesiones parenquimatosas renales entre ambos grupos de pacientes, ni a los 2, ni a los 5 años de seguimiento. En un $20 \%$ de 281 niños con tratamiento quirúrgico y profilaxis antibiótica apareció alguna nueva lesión parenquimatosa renal a lo largo de 5 años de seguimiento, en comparación con el 19,9\% de 291 niños tratados sólo con antibióticos (RR 1,06 [0,77. $1,45])(16)$

Tampoco se encontraron diferencias en términos de progresión de las cicatrices renales ya existentes al diagnóstico, valorados con DMSA, en ambos grupos de pacientes, durante un seguimiento de 10 años. Existe un deterioro de la imagen en 9/107 niños operados y en 13/109 con tratamiento conservador. (RR 0,7 [0,31 a 1,58]). El análisis de subgrupos muestra una relación inversa entre la aparición de nuevas cicatrices y la edad, independientemente del tipo de intervención $(20 \%<2$ años, $10 \%$ entre $2-4$ años, $5 \%<5$ años), siendo los niños menores de dos años los de mayor riesgo (21).

En cuanto al crecimiento global del riñón, tampoco hay diferencias entre ambos grupos, ya que prácticamente todos los pacientes mostraron a los 10 años de seguimiento valores similares de tamaño renal y dentro de parámetros normales (21).

TABLA II. RECOMENDACIONES ESQUEMÁTICAS DE LA SOCIEDAD SUECA DE MEDICINA PARA EL TRATAMIENTO DEL RVU EN NIÑOS SEGÚN EL GRADO DE REFLUJO INICIAL Y SU EVOLUCIÓN (13).

\begin{tabular}{|c|c|c|c|}
\hline $\begin{array}{c}\text { Grado del RVU } \\
\text { inicial }\end{array}$ & $\begin{array}{c}\text { Grado del RVU al } \\
\text { año }\end{array}$ & Tratamiento & Comentarios \\
\hline$|y| \mid$ & & Medidas higiénicas & \\
\hline \multirow[t]{3}{*}{ III y IV } & & Profilaxis antibiótica & \\
\hline & $\mid$ y $\mid$ & Medidas higiénicas & \\
\hline & III y IV & $\begin{array}{l}\text { Niños: medidas higiénicas } \\
\text { Niñas: profilaxis antibiótica } \\
\text { Profilaxis antibiótica }\end{array}$ & $\begin{array}{l}\text { En niñas considerar } \\
\text { corrección quirúrgica o } \\
\text { endoscópica }\end{array}$ \\
\hline V & & $\begin{array}{l}\text { Considerar corrección } \\
\text { quirúrgica }\end{array}$ & $\begin{array}{l}\text { Valorar corrección de } \\
\text { fimosis en niños }\end{array}$ \\
\hline
\end{tabular}


No existe evidencia científica de que la corrección quirúrgica del RVU consiga disminuir la progresión o aparición de nuevas cicatrices renales con respecto a los niños no operados, cuestionando el presumible efecto protector de esta medida terapéutica.

\section{Funcionalismo renal}

Sin lugar a dudas el resultado final que debe hacer ponderar cualquier estrategia terapéutica, es su repercusión sobre el funcionalismo renal. La existencia de lesiones renales bilaterales parece corresponderse con el mayor grado de afectación funcional. Por tanto si bajo ambas estrategias de tratamiento se generan lesiones renales parenquimatosas similares, es de esperar que estos resultados se extrapolen al funcionalismo. La presencia de hipertensión arterial o insuficiencia renal, representan los dos parámetros clave para la valoración inicial del funcionalismo, y su deterioro progresivo a lo largo de los años ha sido bien descrito (23). Las cifras descritas en estudios observacionales son muy variadas, estimándose la presencia de hipertensión arterial en la edad adulta entre un $24-46 \%$ y de disminución del filtrado glomerular entre un $5-25 \%$ en pacientes con antecedentes de nefropatía de reflujo establecida desde la infancia (24). Por el contrario, sus cifras durante la infancia son de mucha menor magnitud, aunque la nefropatía de reflujo sigue ocupando un papel preponderante en los casos de insuficiencia renal crónica terminal. El riesgo de insuficiencia renal crónica terminal secundario a nefropatía de reflujo asociada a lesiones renales bilaterales, se estima en un $9 \%(25)$, aunque los datos reales aportados de registros nacionales varían entre un 0-8\% según las series (26).

Al analizar los datos de los metaanálisis seleccionados vemos que no hay diferencias en cuanto a disminución del filtrado glomerular en el seguimiento de los niños con reflujo, operados o no. En cuatro ensayos clínicos randomizados independientes, que comprenden un total de 196 niños operados y 185 niños con tratamiento conservador, no se objetivaron diferencias en las tasas de filtrado glomerular entre ambos grupos. $(20,27,28,29)$.

\section{TABLA III. COMPARACIÓN DE LOS PRINCIPALES RESULTADOS DEL TRATAMIENTO CONSERVADOR O INTERVENCIONISTA, DEL RVU EN EL NIÑO, SEGÚN DATOS EXTRAÍDOS DE ENSAYOS CLÍNICOS RANDOMIZADOS, INCLUIDOS EN EL METAANÁLISIS DE WHEELER (16).}

\begin{tabular}{|c|c|c|c|}
\hline $\begin{array}{l}\text { Variables ponderadas } \\
\text { (referencia biblio.) }\end{array}$ & $\begin{array}{c}\text { Tratamiento } \\
\text { intervencionista } \\
\text { (casos/total) }\end{array}$ & $\begin{array}{l}\text { Tratamiento } \\
\text { conservador } \\
\text { (casos/total) }\end{array}$ & $\begin{array}{c}\text { Riesgo relativo } \\
\text { (intervalo de confianza } \\
95 \% \text { ) }\end{array}$ \\
\hline ITUs totales a los 5 años $(17,18,30)$ & $\begin{array}{c}36,5 \% \\
(86 / 235)\end{array}$ & $\begin{array}{c}36,8 \% \\
(90 / 244)\end{array}$ & $0,99(0,26-1,35)$ \\
\hline Pielonefritis a los 5 años $(17,30)$ & $\begin{array}{c}9,4 \% \\
(20 / 211)\end{array}$ & $\begin{array}{c}22 \% \\
(48 / 218)\end{array}$ & $0,43(0,27-0,7)$ \\
\hline $\begin{array}{l}\text { Nuevas lesiones renales a los } 5 \\
\text { años }(17,18,20,30)\end{array}$ & $\begin{array}{c}20,7 \% \\
(57 / 275)\end{array}$ & $\begin{array}{c}19,5 \% \\
(58 / 297)\end{array}$ & $1,09(0,79-1,49)$ \\
\hline $\begin{array}{l}\text { Progresión de las lesiones existentes } \\
\text { en } 5 \text { años }(17,18,30)\end{array}$ & $\begin{array}{c}19,6 \% \\
(44 / 224)\end{array}$ & $\begin{array}{c}19,6 \% \\
(48 / 244)\end{array}$ & $0,99(0,69-1,42)$ \\
\hline $\begin{array}{l}\text { Nuevas cicatrices renales de los } 5 \text { a } \\
10 \text { años (21) }\end{array}$ & $\begin{array}{c}12,7 \% \\
(14 / 110)\end{array}$ & $\begin{array}{c}14,1 \% \\
(16 / 113)\end{array}$ & $0,9(0,46-1,75)$ \\
\hline $\operatorname{HTA}(18,20)$ & $\begin{array}{r}6,6 \% \\
(5 / 75)\end{array}$ & $\begin{array}{l}7,5 \% \\
(6 / 79)\end{array}$ & $0,93(0,26-3,42)$ \\
\hline IRCT $(18,20)$ & $\begin{array}{c}4 \% \\
(3 / 75)\end{array}$ & $\begin{array}{c}3,7 \% \\
(3 / 79)\end{array}$ & $1,07(0,23-5,04)$ \\
\hline
\end{tabular}


La incidencia de hipertensión arterial o insuficiencia renal crónica terminal en el seguimiento de ambos tipos de pacientes, fue similar. Analizando dos ensayos clínicos randomizados con seguimiento a 5 años encontramos que tras el periodo de seguimiento, 3 niños de 97 operados entraron en IRCT, y 3 niños de 106 no operados. En cuanto a la aparición de HTA, en estos dos mismos ensayos se detectan 3 casos entre los niños operados y 5 casos en los niños bajo tratamiento conservador $(18,20)$.

Por tanto ambas estrategias son de igual eficacia para preservar el funcionalismo renal, y por tanto parece razonable que la mayoría de las recomendaciones realizadas por sociedades científicas o guías de práctica clínica aboguen por iniciar el tratamiento del RVU dilatado con tratamiento conservador.

Un grupo de especial riesgo lo constituyen los niños con RVU de grado elevado (IV-V) bilateral y cicatrices establecidas. La mayoría de ellos desarrolla una nefropatía de reflujo precoz que condicionará de forma determinante su evolución. En ellos parece razonable que cualquier medida destinada a disminuir el incremento de daño renal adquirido o de infecciones urinarias, pueda estar recomendada. Así en los niños con este grado de reflujo es donde encontramos un mayor grado de actitudes terapéuticas intervencionistas, promoviendo la reparación quirúrgica del reflujo a partir del año de vida (12). Sin embargo, el único ensayo clínico publicado que aborda este tipo de pacientes no encuentra diferencias en la evolución de la nefropatía de reflujo, entre los niños operados y no operados (29). Smellie seleccionó un total de 52 niños, con RVU bilateral de grado elevado y cicatrices establecidas, donde más del $50 \%$ tenían ya una disminución moderada del filtrado glomerular y 2 de ellos hipertensión arterial. Se randomizaron de forma estratificada, según sexo, edad y grado de daño renal, para recibir corrección quirúrgica del RVU o manejo médico conservador. En el seguimiento a 4 y 10 años, no se encontraron diferencias en la evolución del funcionalismo renal entre ambas series, ni en la aparición o progresión de cicatrices renales. Dos niños de cada grupo entraron en insuficiencia renal crónica terminal durante el seguimiento. Sus resultados parecen cuestionar, por tanto una actitud intervencionista, incluso en el subgrupo de pacientes de riesgo más elevado de insuficiencia renal.

\section{REFLEXIONES FINALES}

El número global de infecciones urinarias presentadas por estos pacientes tras largos periodos de observación, es independiente del tratamiento del reflujo realizado, aunque el tratamiento quirúrgico consigue disminuir el número de pielonefritis. La corrección quirúrgica del RVU no consigue disminuir la progresión o aparición de nuevas cicatrices renal, ni alterar el funcionalismo renal, con respecto a los niños no operados, tras periodos prolongados de seguimiento.

De estos datos se deduce que el tratamiento quirúrgico del RVU primario no ofrece más beneficios que el tratamiento conservador y no debe plantearse como tratamiento de primera elección, aunque siempre deberá valo- rarse la opinión de los pacientes y los factores condicionantes individuales.

Las nuevas modalidades de tratamiento intervencionista, por medio de la inyección endoscópica transuretral de macroplásticos, que son mucho menos agresivas, y confieren menor riesgo de eventos adversos, que las técnicas quirúrgicas tradicionales deben ser todavía rigurosamente contrastadas con los métodos de manejo conservador. Esta modalidad es cada vez más simple y posiblemente no incluya de forma reglada la profilaxis antibiótica universal. Deberían realizarse ensayos clínicos aleatorizados que permitan definir nuevas estrategias en un futuro cercano, a la luz de nuevas evidencias científicas. Mientras esto sucede, la evidencia científica actual aconseja un manejo poco agresivo de los niños con RVU.

\section{BIBLIOGRAFÍA Y LECTURAS RECOMENDADAS ("lectura de interés $y^{* *}$ lectura fundamental)}

1. LEBOWITZ, R.L.; OLBING, H.; PARKKULAINEN, K.V. y cols.: "International Reflux Study in Children. International System of radiographic grading of vesicoureteric reflux". Pediatr. Radiol., 15: 105, 1985.

2. SOMNER, J.R.; STHEPHENS, F.D.: "Morphogenesis of nephropathy with partial ureteral obstruction and vesicoureteral reflux". J. Urol., 125: 67, 1981.

3. CHANDRA, M.; MADDIX, H.: "Urodynamic dysfuntion in infants with vesicoureteral reflux". J. Pediatr., 136: 754, 2000.

*4. DILLON, M.J.; GOONASEKERA, C.D.: "Reflux nephropathy". J. Am. Soc. Nephrol., 9: 2377, 1998.

*5. SMELLIE, J.M.; NORMAND, I.C.; KATZ, G.: "Children with urinary infection: A comparison of those with and those without vesicoureteric reflux". Kidney. Int., 20: 717, 1981.

6. CHERTIN, B.; PURI, P.: "Familial vesicoureteral reflux". J. Urol., 169: 1804, 2003.

JODAL, U.: "The natural history of bacteriuria in childhood". Infect. Dis. Clin. North Am., 1: 713, 1987.

*7. ARDISSINO, G.; AVOLIO, L.; DACCO, V. y cols.: "Long-term outcome of vesicoureteral reflux associated chronic renal failure in children. Data from the Italkid project". J. Urol., 172: 305, 2004.

8. UPADHYAY, J.; McLORIE, G.A.; BOLDUC, S. y cols.: "Natural history of neonatal reflux associated with prenatal hydronephrosis: Long-term results of a prospective study". J. Urol., 169: 1837, 2003.

9. GOLDMAN, M.; BISTRITZER, T.; HORNE, T. y cols.: "The etiology of renal scars in infants with pyelonephritis and vesicoureteral reflux". Pediatr. Nephrol., 14: 385, 2000.

10. ARANT, B.S.: "Medical management of mild and moderate vesicoureteral refllux: Follow up studies of infants and young children. A preliminary report of the Southwest Pediatric Nephrology Study Group". J. Urol., 148: 1683, 1992.

**11. ELDER, J.S.; PETERS, C.A.; ARANT, B.S. y cols.: "Pediatric Vesicouteral Reflux Guidelines Panel Summary Report on the management of Primery Vesicoureteral Reflux in children”. J. Urol., 157: 1846, 1997. 
**12. JODAL, U.; JACOBSSON, B.: "Vesico-ureteric reflux in children. Proceedings of a state-of-art symposium organized by the Swedish Medical Research Council 1997”. Acta Paediatr. Suppl., 431, 1999.

13. ELO, J.; TALLGREN, L.G.; ALFTHAN, O. y cols.: "Character of urinary tract infections and pyelonephritic reanl scarring after antireflux surgery". J. Urol., 129: 343, 1983.

14. WHEELER, D.; VIMALACHANDRA, D.; HODSON, E.M. y cols.: "Antibiotic and surgery for vesicoureteric reflux: A meta-analysis of randomised controlled trials". Arch. Dis. Child., 88: 688, 2003.

**15. WHEELER, D.; VIMALACHANDRA, D.; HODSON, E.M. y cols.: "Interventions for primary vesicoureteric reflux". Cochrane Database Syst. Rev., 3: 1532, 2004.

*16. WEISS, R.; DUCKETT, J.; SPITZER, A.: "Results of a arandomized clinical trial of medical versus surgical management of infants and children with grades III and IV primary vesicoureteral reflux (United States)". Journal of Urlogy, 148: 1667, 1992.

*17. SMELliE, J.M.; TAMMINEN, T.; OBLING, H. y cols.: "Five year study of medical or surgical treatment in children with severe reflux: Radiologic findings. The International Reflux Study in Chjildren”. Pediatr. Nephrology., 6: 223, 1992.

*18. DUCKETT, J.W.; DIXON, W.R.; WEISS, R.: "Surgical results: International Reflux Study in children - United States Branch". J. Urol., 148: 1674, 1992.

*19. BIRMINGHAM REFLUX STUDY GROUP.: "Prospective trial of operative versus non-operative treatment of severe vesicoureteric reflux in children: Five years observations". BMJ., 295: 237, 1987.

**20. JODAL, U.; SMELLIE, J.M.; LAX, H. y cols.: “Tenyear results of randomized treatment of children with severe vesicoureteralreflux. Final report of the International Reflux Study in Children”. Pediatr. Nephrol., 21: $785,2006$.
21. VENHOLA, M.; HUTTUNEN, N.P.; UHARI, M.: "Meta-analysis of vesicoureteral reflux and urinary tract infection in children". Scand. J. Urol. Nephrol., 40: 98, 2006.

22. ZANG, Y.; BAILEY, R.R.: "A long term follow up of adults with reflux nephrophathy”. NZ. Med. J., 25: 51, 1995.

23. JACOBSON, S.H.; HANSSON, S.; JAKOBSSON, B.: "Vesico-ureteric reflux: Occurrence and long-term risks". Acta Paediatr. Suppl., 431: 22, 1999.

*24. STARK, H.: "Urinary tract infections in girls: The costeffectiveness of currently recommended investigative routines". Pediatric. Nephrology., 11: 174, 1997.

25. ESBJORNER, E.; BERG, U.; HANSSON, S.: "Epidemiology of chronic renal failure in children: A report from Sweden 1986-1994". Swedish Pediatric. Nephrology Association. Pediatric Nephrology, 11: 438, 1997.

26. CAPOZZA, N.; CAIONE, P.: "Dextranomer-hyaluronic acid copolymer implantation for vesico-urtercic reflux: A randomized comparison with antibiotic prophylaxis". J. Pediatr., 140: 230, 2002.

27. MORRIS, M.C.; ROTHWELL, D.L.; PAYKEL, A.D.: "A prospective study of vesico-ureteral reflux and renal fuction in children". Second CJ Hodson Symposium on Reflux Nephropathy, Christchurch, New Zeland, 1991.

**28. SMELLIE, J.M.; BARRATT, T.M.; CHANTLER, C.: "Medical versus surgical treatment in children with severe bilateral vesicoureteric reflux an bilateral nephropathy: A randomised trial". Lancet., 357: 1329, 2001.

29. JODAL, U.; KOSKIMIES, O.; MASÓN, E. y cols.: "Infection pattern in children with vesicoureteral reflux randomly allocated to operation or long-term antibacterial prophylaxis. The Internacional Reflux Study in Children”. J. Urol., 148: 1650, 1992. 